\title{
Aa. Vv., Stratégies narratives 2: Le roman contemporain, Actes du Colloque de Gênes 14-15 décembre 2001
}

\section{Cecilia Rizza}

\section{OpenEdition}

\section{Journals}

Edizione digitale

URL: https://journals.openedition.org/studifrancesi/37118

DOI: 10.4000/studifrancesi.37118

ISSN: 2427-5856

\section{Editore}

Rosenberg \& Sellier

\section{Edizione cartacea}

Data di pubblicazione: 15 décembre 2004

Paginazione: $572-574$

ISSN: 0039-2944

\section{Notizia bibliografica digitale}

Cecilia Rizza, «Aa. Vv., Stratégies narratives 2: Le roman contemporain, Actes du Colloque de Gênes 14-15 décembre 2001», Studi Francesi [Online], 144 (XLVIIII | III) | 2004, online dal 30 novembre 2015 consultato il 08 mai 2021. URL: http://journals.openedition.org/studifrancesi/37118; DOI: https:// doi.org/10.4000/studifrancesi.37118

Questo documento è stato generato automaticamente il 8 mai 2021.

\section{(c) $($ ) $(9)$}

Studi Francesi è distribuita con Licenza Creative Commons Attribuzione - Non commerciale - Non opere derivate 4.0 Internazionale. 


\title{
Aa. Vv., Stratégies narratives 2: Le roman contemporain, Actes du Colloque de Gênes 14-15 décembre 2001
}

\author{
Cecilia Rizza
}

\section{NOTIZIA}

Aa. Vv., Stratégies narratives 2: Le roman contemporain, Actes du Colloque de Gênes 14-15 décembre 2001, Sous la direction de Rosa Galli Pellegrini, Università degli Studi di Genova, Dipartimento di Lingue e letterature straniere moderne, Schena Editore, Presses de l'Université de Paris Sorbonne, 2003, pp. 358.

1 Nel maggio-agosto 2000 questa rivista pubblicava un numero tematico dedicato allo studio delle stratégies narratives nel romanzo francese dei secoli XVII XVIII e XIX. Presentato da Rosa Galli Pellegrini, autrice anche di un contributo sull'Ibrahim di George de Scudéry il fascicolo raccoglieva i primi frutti di una ricerca svoltasi nell'ambito della Sezione di Francesistica del Dipartimento di Lingue e letterature straniere dell'Università di Genova. Il discorso allora iniziato coniugava, sia pure con qualche libertà, la lezione dello strutturalismo e del formalismo con la metodologia critica suggerita dalle opere di Gérard Genette, Jean Starobinski, Philippe Lejeune, Umberto Eco (per non citare che alcuni dei più noti studiosi che si sono occupati del problema), privilegiando l'analisi del rapporto di collaborazione tra autore e lettore. Era pertanto ovvio e direi quasi necessario che a quel primo approccio rivolto ad opere del passato, a secoli in cui talvolta il romanzo era ancora alla ricerca di un proprio statuto, seguisse una riflessione critica interamente dedicata alle strategie narrative nel romanzo francese contemporaneo, a quei romanzi in cui il cosiddetto pacte auctorial diventa più evidente e significativo. I frutti di tale ricerca coordinata da Rosa Galli 
Pellegrini sono stati esposti in un Colloquio di cui escono oggi in volume gli Atti. Ai collaboratori già presenti nel succitato fascicolo, si sono aggiunti, in questa occasione, alcuni autori e studiosi francesi di alto livello e un gruppo di docenti e ricercatori dell'Università di Cagliari che hanno rivolto il loro interesse al romanzo beur.

Il compito di aprire il Colloquio è stato affidato ad Alain Nadaud nella sua veste di autore che a lungo ha dibattuto i problemi della scrittura romanzesca. Ripercorrendo le varie fasi del processo creativo di alcuni suoi romanzi, il Nadaud vi riconosce, oggi, a distanza di tempo, un denominatore comune nelle vicende del parigino maggio '68. Non si tratta della trasposizione, consapevolmente ricercata e diversamente sviluppata in chiave romanzesca, di un'esperienza di vita, bensì di una sorta di secondo livello di lettura, difficilmente riconoscibile se non riferito a quella particolare temperie politica. E proprio questo secondo livello che diventa un second degré de l'écriture è più facilmente percepibile quando, all'esperienza di vita, si sostituisca un testo che ricrea o meglio immagina, inventa quella vita. È quanto mette in luce il contributo di Dominique Rabaté attraverso l'analisi di una serie di autobiographies imaginaires, sorta di gioco grazie al quale la création de l'autre sert à la fois de limite fascinante et de repoussoir. Lo stesso procedimento deliberatamente scelto può riguardare l'autobiografia, come nel caso di Annie Ernaux, qui illustrato da Antonella Arrigoni e ancora più esemplarmente nel $W$ ou le souvenir d' enfance di George Perec cui si riferisce l'analisi acuta e convincente di Daniel Sipe.

Il gioco intelletualistico diventa più evidente se si ha a che fare con un testo letterario preciso; tale è il riferimento all'Astrée proposto da Michel Chaillou di cui si è occupata Chiara Rolla. In tutti questi casi la stratégie narrative di ciascun autore appare implicita e risultano ancora presenti l'esperienza e l'esempio del nouveau roman di cui rappresentano un ulteriore corollario. E proprio ai migliori esiti del nouveau roman si richiama Claude Simon nelle Géorgiques cui Luc Fraisse dedica un saggio particolarmente convincente. Al di là del possibile riferimento al mito di Orfeo (ancora una volta una scrittura au second degré) il critico vi riconosce il processo stesso della creazione romanzesca, chiave di lettura nascosta che fa appello alla capacità del lettore di riconoscerla.

4 Talvolta l'autore, nel suo rapporto con il lettore, sembra divertirsi a brouiller les pistes, com'è il caso di Jean Echenor, in un gioco che - osserva giustamente Cristina Bernazzoli - non vuol essere gratuito, ma obbliga il destinatario dei suoi romanzi a mettersi a sua volta in discussione e a riflettere per scoprire quanto di problematico, sia a livello di scrittura, sia in rapporto con la realtà, si cela nella sua opera. In alcuni romanzi (è il caso di Roman roi e di Roman furieux di Renaud Camus, cui dedica la sua attenzione Cecilia Torelli) le allusioni e la scoperta di significati nascosti in cui il lettore si trova impegnato può alla lunga risultare fine a se stesso e, come tutti i giochi, apparire gratuito e quindi futile sicché il lettore non avvertito o intellettualmente preparato può abbandonarne la lettura. In altri casi l'appello al lettore diventa esplicito, grazie all'uso di paratextes che lo chiamano direttamente in causa. Un esempio dei più significativi $\mathrm{e}$ scoperti ci è dato da Le livre des nuits e da L'enfant médusé in cui, come sottolinea Elisa Bricco, Sylvie Germain, attraverso i titoli dei capitoli, i loro incipit e altre forme di avant-textes induce e quasi obbliga in qualche modo il lettore a riconoscere la struttura stessa della narrazione.

5 In questa prospettiva acquista particolare interesse lo studio degli incipit. Difficile è fissare i limiti di incipit nel significato tradizionale del termine: una frase iniziale? una 
pagina? un intero capitolo? Più facile riconoscerne la duplice funzione: da un lato esso stabilisce un patto iniziale dell'autore con il suo lettore; dall'altra esso svolge una funzione strategica essendo il luogo in cui l'autore fornisce al lettore un certo numero d'informazioni utili ad introdurlo nel mondo creato dalla finzione romanzesca. Come osserva Rosa Galli Pellegrini a conclusione del suo contributo sull'opera di Pierre Michon, entrambe queste funzioni presuppongono e per la prima sarebbe meglio dire esigono una complicità tra autore e lettore possibile soltanto se e quando essi appartengono ad una stessa cultura: De cette façon l'on procède à la sélection d'un public élitaire duquel seront exclus ceux qui ne sont pas en mesure d'accepter les règles du jeu. Ma ciò vale anche per quei romanzi cui è dedicata l'ultima parte del volume i quali pur nella modestia del loro valore letterario denunciano l'appartenenza ad una specifica identità culturale, non facilmente accessibile a tutti anche a causa della lingua beur cui attingono.

La varietà dei contributi pubblicati in questo volume e raccolti per lo più e volutamente seguendo l'ordine alfabetico dei cognomi degli studiosi chiamati a collaborare, concorre a postulare una visione coerente ed univoca della narrativa francese contemporanea. Essa si definisce come una riflessione sull'atto di scrivere, estranea ormai o comunque indifferente ad ogni implicazione psicologica o sociologica o politica e quindi priva di un qualunque messaggio che non sia autoreferenziale. È certo che a questo risultato conduce, direi quasi necessariamente, la scelta metodologica e l'ambito stesso entro il quale la ricerca si è svolta. Essa riguarda infatti esclusivamente il romanzo francese contemporaneo; difficilmente la si potrebbe applicare ad altre opere narrative del nostro tempo e comunque aprirebbe problematiche diverse. Penso in particolare all'attuale produzione dei grandi autori dell'America latina, ma anche ai nostri Tabucchi, Mazzantini ecc. Per contro non va trascurato il fatto che questa forma di narrazione corrisponde in qualche modo ad analoghi esiti cui sono giunte le arti figurative e certa musica del nostro tempo. Semmai è lecito domandarsi se è giusto continuare a definire romanzo questa forma di scrittura e quale sviluppo essa potrà avere in futuro.

7 Per questo, a conclusione di un così importante e originale contributo critico cui aggiunge autorevolezza la specifica bibliografia, mi sembra soprattutto pertinente l'ampio saggio di Sergio Poli: Internet, le pacte auctorial et l'avenir du roman. Tracciando una rapida storia del genere narrativo lo studioso ne fa coincidere la fortuna con l'imporsi di un certo tipo di società che potremmo definire borghese e la collega a quello straordinario strumento di diffusione che fu l'invenzione della stampa. La crisi d'identità che attraversa oggi il romanzo sarebbe in relazione con le profonde trasformazioni della nostra cultura e civiltà cui ha prepotentemente contribuito l'avvento di nuove tecniche di comunicazione di massa. L'avvenire di questo genere letterario si troverà forse in internet, nella creazione interattiva di opere in cui più stretto e simultaneo si fa il tradizionale rapporto tra autore e lettore, dal momento che l'uno e l'altro sono chiamati a concorrere in tempo reale alla loro creazione. 